\title{
Combined effect of moisture and electrostatic charges on powder flow
}

\author{
Antonella Rescaglio ${ }^{1}$, Julien Schockmel ${ }^{1}$, Nicolas Vandewalle ${ }^{1}$, and Geoffroy Lumay ${ }^{1, \star}$ \\ ${ }^{1}$ CESAM-GRASP, University of Liége, Belgium
}

\begin{abstract}
It is well known in industrial applications involving powders and granular materials that the relative air humidity and the presence of electrostatic charges influence drastically the material flowing properties. The relative air humidity induces the formation of capillary bridges and modify the grain surface conductivity. The presence of capillary bridges produces cohesive forces. On the other hand, the apparition of electrostatic charges due to the triboelectric effect at the contacts between the grains and at the contacts between the grains and the container produces electrostatic forces. Therefore, in many cases, the powder cohesiveness is the result of the interplay between capillary and electrostatic forces. Unfortunately, the triboelectric effect is still poorly understood, in particular inside a granular material. Moreover, reproducible electrostatic measurements are difficult to perform. We developed an experimental device to measures the ability of a powder to charge electrostatically during a flow in contact with a selected material. Both electrostatic and flow measurements have been performed in different hygrometric conditions. The correlation between the powder electrostatic properties, the hygrometry and the flowing behavior are analyzed.
\end{abstract}

\section{Introduction}

When two materials are rubbed, electric charges are exchanged at the surfaces $[1,2]$. This contact electrification is one of the oldest scientific subject. Thales of Miletus (624 - 546 BC) discovered that amber and wool are electrified when rubbing together. It is interesting to note that the word 'electron' comes from the greek word for 'amber' and the term 'triboelectric' means 'rubbing amber' in greek. Despite the numerous studies dedicated to this subject, the fundamental mechanisms behind this triboelectric effect is not fully understood. The charging of objects composed by the same material [3] and charging of powders $[2,4,5]$ are two examples of poorly understood subject. Even the basic question related to the nature of the transferred charges (electrons, ions or material) is still debated. The difficulties are related to the non-equilibrium character of the tribolectectric dynamic and to the variety of mechanisms behind this effect. Different mechanisms could induce triboelectric charges inside a granular material: impact, rolling and sliding (or rubbing) [6].

On the one hand, the triboelectric effect is useful for many applications (electrophotography, powder coating processes, triboelectric separators, ...), but on the other hand, the triboelectrification causes complications. The apparition of electric charges inside a granular material can produce sparks and cause explosion hazards in dispersed fine particles. In pharmaceutical industries, the contact charging during particles flows induces agglomeration and segregation witch cause dosage problems.
In combination with electrostatic charges, moisture is known to affect both static and dynamic behaviors of a granular materials [7-9]. However, the effect of moisture is far to be obvious. Indeed, moisture influences both surface grains conductivity and capillary bridges formation. For low relative air humidity, the electrical conductivity necessary for charge dissipation is reduced and the relative permittivity comes close to vacuum permittivity. Therefore, the electric charges created by triboelectric effects lead to uncontrolled electric field, electrostatic forces between the grains and/or between the grains and the container. For high relative air humidity, the electrical conductivity increases, liquid bridges may be formed at the contacts between the grains and the relative air permittivity increases. Therefore, the electrical charges are dissipated more easily. However, the apparition of liquid bridges induces cohesive forces inside the packing [10]. At intermediate relative humidity the cohesion is expected to decrease. In addition to these effects, the humidity could also modify the friction due to lubrication effects [11].

In the present paper, we describe an experimental method to measure the quantity of electric charges created inside a powder during a bulk flow in contact with a selected material. This device is called a powder triboelectrometer. In addition to the electrostatic measurement, a flow measurement based on the classical rotating drum method is used to study the influence of the relative air humidity on powder flow. In particular, we show how the association of capillary and electrostatic forces modifies the macroscopic properties of powders. Finally, we discuss how the results can be used to optimize industrial applications involving powders like pneumatic conveying.

\footnotetext{
^e-mail: geoffroy.lumay@ulg.ac.be
} 

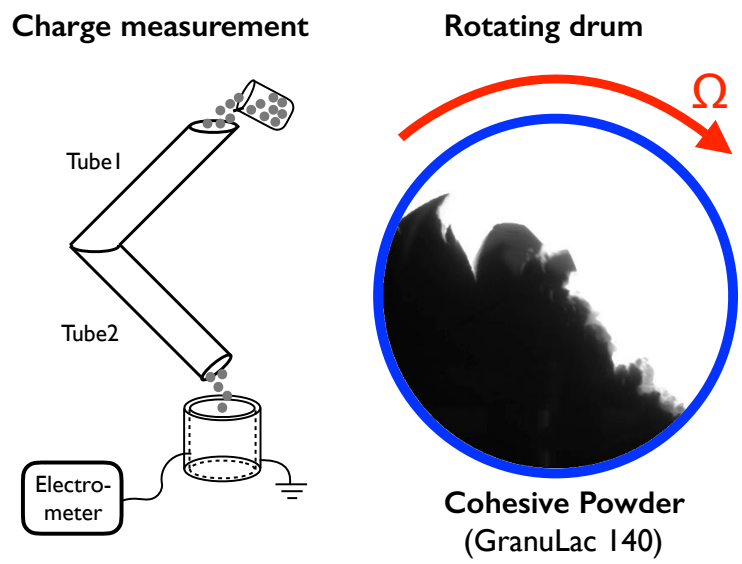

Figure 1. Sketch of the experimental set-ups used to perform the measurements. (left) The powder triboelectrometer measures the electrostatic charge inside the powder after a flow in contact with a selected material. (right) The powder flowing properties are measured in a rotating drum.

\section{Set-up}

\subsection{Powder triboelectrometer}

Figure 1 (left) shows the experimental set-up used to measure the total electrostatic charge created inside a granular material during a flow in contact with a selected material. The sample is poured manually (the feeding could be automated) in a V-tube and flows to a Faraday cup. The Vtube is an assembly of two tubes (Tube1 and Tube2) of length $\mathrm{L}=350 \mathrm{~mm}$ and internal diameter $\mathrm{D}=47 \mathrm{~mm}$ to form a $\mathrm{V}$ shape with an angle of $90^{\circ}$. The tube material can be selected for each tube. In the present study, we used 316L stainless steel (S.S.) and ABS. The faraday cup is connected to a customized electrometer able to measure electrostatic charges ranging from $0.1 \mathrm{nC}$ to $1 \mu \mathrm{C}$. At the end of the flow, the total value of the electric charge $\mathrm{Q}$ present in the powder is measured and the charge density $Q / m$ is computed, where $m$ is the sample mass, is measured.

The V-tube geometry has been selected to combine the different mechanisms leading to tribo-electrification: (i) friction between the grains, (ii) friction between the grains and the wall and (iii) impacts of the grains on the wall at the connection between the two tubes.

The device used to perform the present study is the prototype of the GranuCharge presently commercialized by GranuTools.

\subsection{Flow measurement}

Experimentally, the most practical geometry to study a free surface flow of granular materials is the rotating drum. This flow geometry has been extensively studied with non cohesive granular materials and with cohesive powders [12-14]. In the case of cohesive grains, the flow is intermittent in the whole range of rotating speeds.
An aluminum cylinder of internal diameter $D=84 \mathrm{~mm}$ and length $L=20 \mathrm{~mm}$ with glass side walls is half-filled with the powder. The cylinder rotates around its axis at an angular velocity $\Omega$ producing the flow of the grains (see Figure 1 (right)). The rotating speed $\Omega=8 R P M$ has been selected to obtain a roughly flat interface without fluctuation with a non-cohesive granular material. Therefore the fluctuations of the interface obtained with a cohesive powder is related to the existence of cohesive forces. From 50 images of the rotating powder bed separated by $0.5 \mathrm{~s}$, the average position of the powder/air interface and the fluctuations (standard deviation) around this average interface are calculated. The standard deviation is integrated over the whole interface to obtain the total interface fluctuation $\sigma[15,16]$.

The device used to perform the present study is the prototype of the GranuDrum presently commercialized by GranuTools.

\subsection{Conditioning}

Before the flowability measurement in the rotating drum, the powder is conditioned during a time $T$ in a slowly rotating reactor. The reactor is a $316 \mathrm{~L}$ stainless steel tube of internal diameter $D=48 \mathrm{~mm}$ and length $L=320 \mathrm{~mm}$. The tube axis is placed horizontally. The tube is rotating around its axis at 0.66 RPM. A flux $F=0.3 \mathrm{l} / \mathrm{min}$ of air with controlled relative humidity flows through the reactor. The slow motion of the reactor allows the humid air to penetrate inside the bulk. The accessible values of the relative humidity range from $\mathrm{RH}=0 \%$ to $\mathrm{RH}=95 \%$. Directly after this preparation, the drum cell is half filled by the powder and is closed hermetically.

\subsection{Powders}

Two lactose powders produced by the company Meggle and widely used in pharmaceutical industries have been selected: GranuLac 140 (Lactose1) and InhaLac 230 (Lactose2). Granulac 140 powder comes from milling process with an average grains diameter of $65 \mu \mathrm{m}$ and a standard deviation of $55 \mu \mathrm{m}$ (see insert of Figure 2). Inhalac 230 powder is a milled and sieved lactose powders with an average grains diameter of $98 \mu \mathrm{m}$ and lower standard deviation of $39 \mu \mathrm{m}$. We received the samples in small sealed bags in order to perform the measurements without powder aging.

\section{Results and discussion}

A typical flow of lactose grains at the V-tube output is shown by Figure 2. The grains are electrostatically charged and are repealing each other.

Figure 3 shows the charge density $Q / m$ obtained with the lactose powders for different relative air humidities and for different materials in contact (Stainless steel and ABS). The different relative air humidity values have been obtained by switching off the humidity regulator of the laboratory. Therefore, we took advantage of the Belgian meteorological fluctuations. The sign of the charge depends 


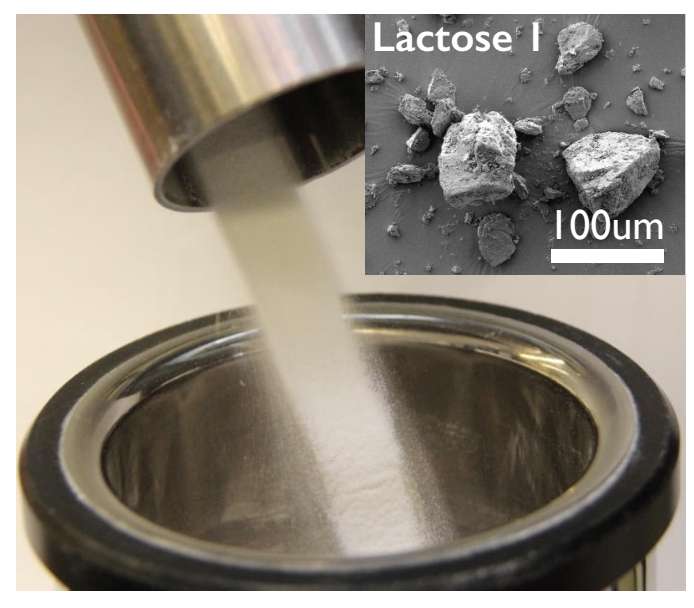

Figure 2. Flow of a lactose powder between the V-tube output and the Faraday cup. The insert shows a micrograph of a few lactose (GranuLac140 from Meggle) grains.

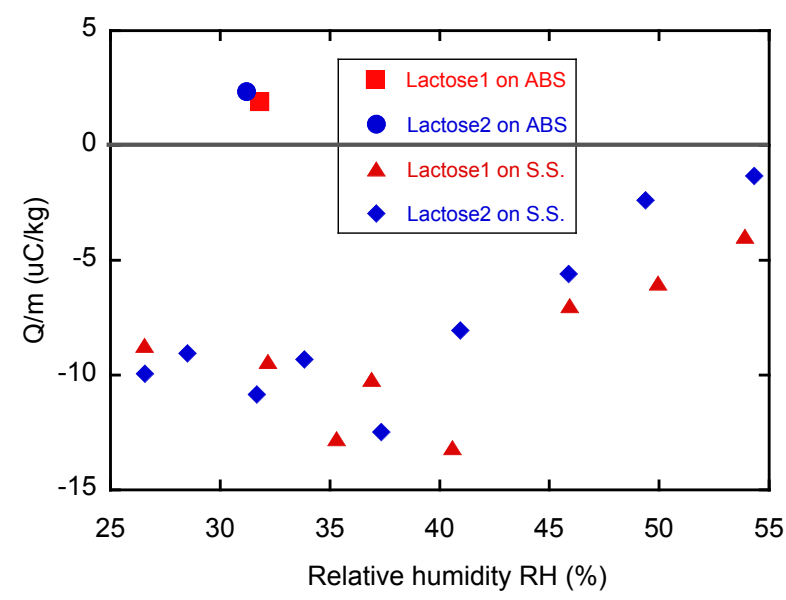

Figure 3. Charge density $Q / m$ obtained with the powder triboelectrometer for different relative air humidities and for different materials in contact (Stainless steel and ABS)

on the material in contact: positive for lactose on $\mathrm{ABS}$ and negative for lactose en stainless steel. Therefore, in a triboelectric series, lactose powders should be placed between stainless steel and ABS. Moreover, the absolute value of the charge density decreases drastically when the relative air humidity goes from $40 \%$ to $50 \%$. This observation is in agreement with the results obtained very recently by Schella et al [17] in a shaken cell.

In order to study the influence of relative air humidity on powder cohesiveness, the samples of powder has been conditioned at fixed relative air humidity during $T=150$ minutes before filling the rotating drum. The cohesiveness is found to decrease slightly in the range of relative humidity from $0 \%$ to $40 \%$ (see Figure 4 ). Therefore, the cohesive forces inside the bulk decrease and the flowability is enhanced. Afterward, the cohesion increase importantly from $\mathrm{RH}=50 \%$ to $\mathrm{RH}=100 \%$. Then, the relative humidity that optimizes the flowability of lactose grains is situated between $30 \%$ and $50 \%$. For low humidity con-

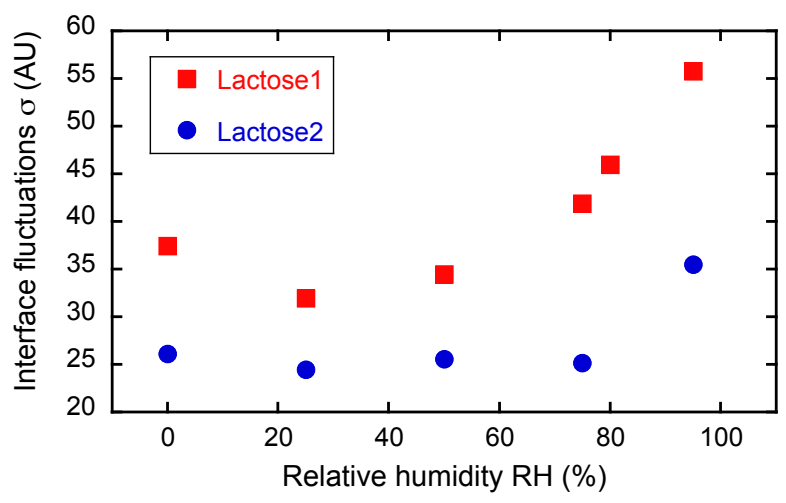

Figure 4. Fluctuations $\sigma$ of powder/air interface measured in the rotating drum at a rotating speed $\Omega=8 \mathrm{RPM}$ as a function of the relative humidity $R H$ for a residence time in the conditioner fixed to $T=150$ minutes.

ditions, the cohesion is induced by the apparition of electric charges on the surface of powder grain (see Figure 3). For the extremal case $\mathrm{RH}=0 \%$ the powder was sticking on the drum surfaces due to these electrostatic charges. For high humidity conditions, the condensation of water at the surface of the grains leads presumably to the formation of capillary bridges at the contact between the grains, increasing powder cohesiveness. For the relatively short residence times in high humidity conditions considered in the present study, we do not expect water absorption inside the grains.

Globally, the cohesiveness of Lactose 1 is higher than the cohesiveness of Lactose 2 (see Figure 4) in the whole range of RH. Indeed, the average grain size is lower in Lactose1. Therefore, the ratio between the cohesive forces acting on a grain and the grain weight is higher in Lactose1.

During powder pneumatic transport, it is well known in industries that the pipe material influences the apparition of electrostatic charges. Stainless steel is usually used. Some parts (bends, flexible connections, ...) are sometimes made in plastics. The problems due to the apparition of electrostatic charges are difficult to predict and the approach is mainly empirical. The measurement device considered in the present paper can be used to predict the apparition of electrostatic charge in these devices. Moreover, the association of different materials can be considered. The measurement with lactose powders has been made in four configurations: two S.S. tubes, two ABS tubes, an ABS tube followed by a S.S. tube and a S.S. tube followed by an ABS tube. Figure 5 shows that the tube order plays an important role on the final charge. In one configuration (S.S. tube followed by an ABS) the final charge is reduced. Moreover, the final charge is not a simple combination of the charge obtained separately with S.S. and ABS. The non-linearity of the charging mechanism and the difference of flowing speed in the two tubes could explain this difference. 


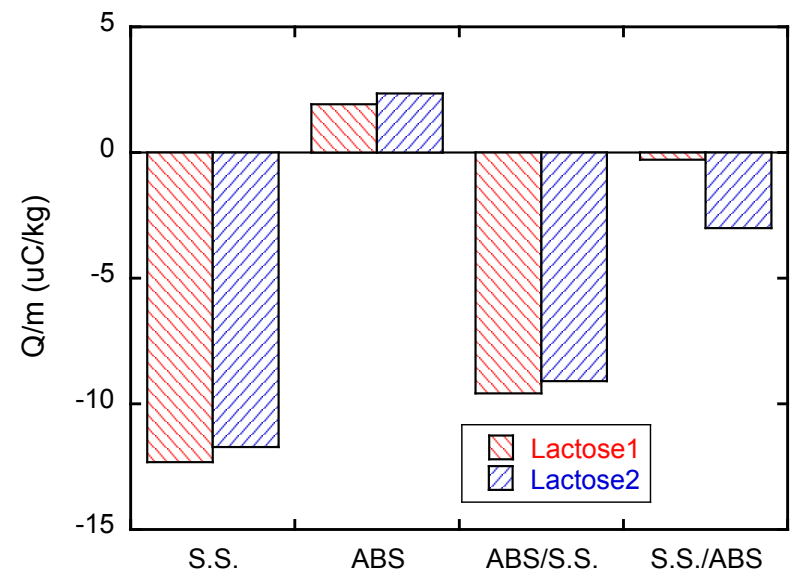

Figure 5. Charge density $Q / m$ obtained with the powder triboelectrometer in four tubes configurations: two S.S. tubes, two ABS tubes, an ABS tube followed by a S.S. tube and a S.S. tube followed by an ABS tube.

\section{Conclusion}

We have shown that the relative air humidity affects strongly the flow of powders. The powder cohesiveness increases for both dry and wet conditions. When the air relative humidity is low, grain-grain friction inside the flow induces the appearance of electrical charges in the packing, inducing cohesive forces between the grains. The condensation in high humidity conditions implies the formation of liquid bridges between contacting grains which induce also cohesive forces. Taking both effects (triboelectricity and capillary) into account, the cohesion is minimized for intermediate values of the relative humidity, i.e. between $\mathrm{RH}=30 \%$ and $\mathrm{RH}=50 \%$.

The powders triboelectic properties have been measured with a device formed by a V-tube and a Faraday cup. The measurement has been repeated for different hygrometric conditions and for different materials (stainless steel and ABS) in contact with the powder. The electrostatic charge created inside a powder during a flow decrease drastically when the relative hair humidity becomes higher than $40 \%$. This observation is coherent with the flow behavior.

Finally, we have shown that the association of different materials in a device can reduce the charge acquired by the powder after a flow inside the device.

\section{Acknowledgements}

We Thanks Walloon region (Fonds de maturation - convention 1318086 and BEWARE - convention 1410265) for the financial support.

\section{References}

[1] D. J. Lacks, Nature Physics 6, 324 (2010)

[2] S. Matsusaka, H. Maruyama, T. Matsuyama, and M. Ghadiri, Chemical Engineering Science 65, 5781 (2010)

[3] S. R. Waitukaitis, V. Lee, J. M. Pierson, S. L. Forman, and H.M. Jaeger, Phys. Rev. Lett. 112218001 (2014)

[4] P. M. Ireland, and K. Nicholson, Minerals Eng. 24, 914 (2011)

[5] E. Mersch, G. Lumay, F. Boschini, and N. Vandewalle, Phys. Rev. E 81, 041309 (2010)

[6] P. M. Ireland, Powder Technol. 198, 189 (2010)

[7] J. E. Fiscina, G. Lumay, F. Ludewig, and N. Vandewalle, Phys. Rev. Lett. 105, 048001 (2010)

[8] E. Emery, J. Oliver, T. Pugsley, J. Sharma, and J. Zhou, Powder Technol. 189, 409 (2009)

[9] N. Vandewalle, G. Lumay, F. Ludewig, and J. E. Fiscina, Phys. Rev. E 85, 031309 (2012)

[10] L. Bocquet, E. Charlaix, S. Ciliberto, and J. Crassous, Nature 396735 (1998)

[11] Q. Xu, A. V. Orpe, and A. Kudrolli, Phys. Rev. E 76, 031302 (2007)

[12] J. Rajchenbach, Phys. Rev. Lett. 65, 2221 (1990)

[13] R. Fischer, P. Gondret, and M. Rabaud, Phys. Rev. Lett. 103, 128002 (2009)

[14] A. Castellanos, J.M. V., A.T. Perez, A. Ramos and P.K. Watson, Phys. Rev. Lett. 82, 1156 (1999)

[15] G. Lumay, F. Boschini, K. Traina, S. Bontempi, J.-C. Remy, R. Cloots, and N. Vandewalle, Powder Technology 224, 19 (2012)

[16] F. Boschini, V. Delaval, K. Traina, N. Vandewalle, and G. Lumay, Int. J. of Pharmaceutics 494, 312 (2015)

[17] A. Schella, S. Herminghaus, and M. Schroter, arXiv:1609.04639 (2016) 